\title{
HORIZONTAL CHORDS OF THE GRAPH OF A CONTINUOUS FUNCTION
}

\author{
UWE HERZBERG
}

ABSTRACT. The continuous function $f$, defined on $[a, b]$, changing sign exactly $n$ times on $(a, b)$, and $f(a)=f(b)=0$, has horizontal chords of every length $h<H$ with endpoints in $(a, b)$. We determine the largest such $H$ as a function of $n$.

By a theorem of R. J. Levit [1], we know that if $f$ is a continuous real function on the closed interval $[a, b]$, changes sign exactly $n$ times in the open interval $(a, b)$, and $f(a)=f(b)=0$, then the graph of $f$ has horizontal chords of every length $\left.h \leq H_{1}=(b-a) /[(n+3) / 2)\right]$ with endpoints in $[a, b]$, i.e., for every such $h$ at least one $x \in[a, b-b]$ exists such that $f(x+h)=$ $f(x)$. In case of endpoints in $(a, b], H_{1}$ reduces to $H_{2}=(b-a) /[(n+5) / 2]$. These two bounds cannot be improved.

It is easy to see that $H_{2}$ remains the best possible bound if the endpoints are in $[a, b)$. So far the bounds hold for $n$ both even and odd. The purpose of this note is to consider the situation when we ask for horizontal chords with endpoints in $(a, b)$.

Theorem. If $f$ is a continuous real function on $[a, b]$, changes sign exactly $n$ times in $(a, b)$, and $f(a)=f(b)=0$, then for any positive $h$ such that

$$
h \leq H_{2}=\frac{b-a}{[(n+5) / 2]}=\frac{b-a}{(n+4) / 2} \quad \text { for } n \text { even }(n>0) \text {, }
$$

and

$$
h \leq H_{3}=\frac{b-a}{[(n+7) / 2]}=\frac{b-a}{(n+7) / 2} \text { for } n \text { odd, }
$$

there is a number $x \in(a, b-h)$ such that $f(x+h)=f(x) . H_{2}$ and $H_{3}$ cannot be improved.

Received by the editors May 29, 1974.

AMS (MOS) subject classifications (1970). Primary 26A06; Secondary 39A05.

Key words and phrases. Horizontal chord, change of sign, zero. 
Proof. 1. The following three cases, (a), (b), and (c), hold for $n$ even as well as for $n$ odd.

(a) If $f(a+h) \neq 0$ and $f(b-h) \neq 0$, the statement follows at once from Levit's theorem, as it does in the cases:

(b) $f(a+h)=0, f(b-h) \neq 0$; and

(c) $f(a+h) \neq 0, f(b-h)=0$.

We remark that up to this point for every $h \leq(b-a) /[(n+5) / 2]$, there is a number $x \in(a, b-h)$ such that $f(x)=f(x+h)$.

2. Let $f(a+h)=f(b-h)=0$, and let $n^{*}$ be the number of times $f$ changes sign in $(a+h, b-h)$; then for both even and odd $n$

$$
h \leq(b-a) /[(n+7) / 2] \Leftrightarrow h\{[(n+3) / 2]+2\} \leq b-a .
$$

Hence,

$$
h \leq \frac{b-a-2 h}{[(n+3) / 2]} \leq \frac{(b-h)-(a+h)}{\left[\left(n^{*}+3\right) / 2\right]},
$$

and according to Levit's theorem there is a number $x$ such that

$$
a<a+b \leq x \leq b-2 h<b-h,
$$

i.e., $x \in(a, b-h)$ anyway, and $f(x+b)=f(x)$. For $n$ odd the first part of the Theorem is proved, while for $n$ even we still have to improve the bound $(b-a) /[(n+7) / 2]$, gained under the assumption $f(a+b)=f(b-h)=0$.

3. Let $n$ be even and $n^{*} \leq n-2$. Thus,

$$
h \leq \frac{b-a}{[(n+5) / 2]} \Leftrightarrow h \leq \frac{b-a-2 h}{[(n+1) / 2]},
$$

and since $n^{*} \leq n-2$, it follows that

$$
h \leq \frac{(b-h)-(a+h)}{\left[\left(n^{*}+3\right) / 2\right]} \text {. }
$$

The existence of the required number $x$ is again a consequence of Levit's theorem.

4. Before we continue, let us give a definition. We say that $f$ has a true zero in $x$ if $f$ has a zero in $x$, but does not change sign in $x$.

Let $n$ be even. Assuming $n^{*}=n$ or $n^{*}=n-1$, we still have to prove our statement for the following five cases, respective to the properties of $f$;

(a) $n^{*}=n$, and true zeros in $a+h$ and $b-h$;

(b) $n^{*}=n-1$, true zeros in $a+h$ and $b-h$, and one change of sign in $(a, a+b)$

(c) $n^{*}=n-1$, true zeros in $a+h$ and $b-h$, and one change of sign in $(b-h, b)$;

(d) $n^{*}=n-1$, true zero in $b-h$, and a change of sign in $a+b$; 
(e) $n^{*}=n-1$, true zero in $a+h$, and a change of sign in $b-h$.

We remark that in (a), (d), and (e), $f(x) \geq 0[f(x) \leq 0$, respectively $]$ for every $x \in(a, a+b) \cup(b-h, b)$, and in (b) and (c), $f(x) \geq 0[f(x) \leq 0$, respectively] in one of the intervals $(a, a+b)$ or $(b-h, b)$, while in the other one $f$ changes sign.

In the following two lemmas we assume without loss of generality the case not in brackets in the preceding paragraph.

Lemma 1. If $f$ satisfies (a), (d), or (e), then for every positive $h<$ $(b-a) / 2$ there exists a number $x \in(a, b-h)$ such that $f(x+h)=f(x)$.

Proof of Lemma 1. Suppose for some $h<(b-a) / 2$ such $x$ does not exist. Then for $x \in(a, b-b)$, either (i) $f(x)<f(x+b)$ or (ii) $f(x)>f(x+h)$ is true. If (i) holds there is an integer $m>0$ satisfying $((b-b)-m b) \epsilon$ $(a, a+b)$. It follows that

$$
0=f(b-h)>f((b-h)-m h) \geq 0,
$$

which is a contradiction. If (ii) holds, there is an integer $m>0$ satisfying $((a+b)+m b) \in(b-h, b)$. Thus, the contradiction

$$
0=f(a+b)>f((a+b)+m b) \geq 0
$$

completes the proof of Lemma 1.

Lemma 2. If $f$ satisfies (b) or (c) - without loss of generality we choose (b) - then for every positive $h<(b-a) / 2$ there is a number $x \in(a$, $b-h)$ such that $f(x+h)=f(x)$.

Before starting the proof we give a short summary of the properties of the used function $f$. For $x \in(b-h, b) f(x) \geq 0$ holds; $f$ changes sign-at $x_{0} \in(a, a+h) ; f(x) \geq 0$ for $x \in\left(a, x_{0}\right]$ since $n$ is even, consequently, $f(x)$ $\leq 0$ for $x \in\left[x_{0}, a+h\right]$, and since $f$ has a true zero in $a+h, f(x) \leq 0$ for $x \in\left[x_{0}, a+b+\epsilon\right)$, where $\epsilon>0$.

Proof of Lemma 2. Suppose $h<(b-a) / 2$ and the required $x$ does not exist. It follows that either $f(x)<f(x+h)$ or $f(x)>f(x+b)$ for $x \in(a$, $b-h)$. Let $f(x)<f(x+h) ; f(x) \geq 0$ for $x \in\left(a, x_{0}\right]$ implies $f(x+h)>0$ for $x+h \in\left(a+h, x_{0}+h\right]$. This is a contradiction to the fact $f(x) \leq 0$ for $x \epsilon$ $(a+b-\epsilon, a+h+\epsilon)$, where $\epsilon>0$, shown in the summary above. If $f(x)>$ $f(x+h)$, the proof is the same as in Lemma 1 .

Let $n>0$, then

$$
\frac{b-a}{(n+4) / 2}<\frac{b-a}{2}
$$


According to the two lemmas, this completes the proof of the first part of the Theorem for $n$ even.

5. To see that $H_{j}, j=2,3$, are the best possible bounds, we present two polygonal arcs $R_{j}$ ( $R_{2}$ was already used by Levit) satisfying the conditions of our Theorem and with the property that for any integer $n>0$ and any $H>H_{j}$, there is a number $h<H$ such that $R_{j}(x+h)-R_{j}(x) \neq 0$ for every $x \in(a, b-h)$.

We choose

$$
h \in\left(H_{j}, \min \left\{H_{j-1}, H\right\}\right)
$$

set $m=[(b-a) / h], a_{i}=a+i h, b_{i}=b-i h(i=0,1, \ldots, m)$, and $b_{i}^{\prime}=$ $b_{0}^{\prime}-i h(i=1,2, \cdots, m)$, where

$$
b_{0}^{\prime}=b-w h /(m+1) \text { and } w=(b-a) / h-m .
$$

Thus, the numbers $a_{i}, b_{i}$, and $b_{i}^{\prime}$ form finite sequences

$$
a=a_{0}<b_{m}<a_{1}<\cdots<b_{1}<a_{m}<b_{0}=b
$$

and

$$
a=a_{0}<b_{m}^{\prime}<a_{1}<b_{m-1}^{\prime}<\cdots<a_{m}<b_{0}^{\prime}<b
$$

We proceed with our construction by defining points

$$
\begin{aligned}
& P_{0}(a, 0), \quad P_{i}\left(a_{i}, i-1\right), \quad i=1,2, \cdots, m, \\
& Q_{m}(b, 0), \quad Q_{i}\left(b_{m-i}, 1+i-m\right), \quad i=0,1, \cdots, m-1, \\
& Q_{i}^{\prime}\left(b_{m-i}^{\prime}, i-m-w /(1-w)\right), \quad i=0,1, \cdots, m,
\end{aligned}
$$

and polygonal arcs

$$
\begin{aligned}
& R_{2}(x): P_{0} Q_{0}^{\prime} P_{1} Q_{1}^{\prime} \cdots Q_{m-1}^{\prime} P_{m} Q_{m}^{\prime} Q_{m} \text { for } n \text { even, and } \\
& R_{3}(x): P_{0} Q_{0} P_{1} Q_{1} \cdots Q_{m-1} P_{m} Q_{m} \quad \text { for } n \text { odd. }
\end{aligned}
$$

Since each of the points $Q_{i}^{\prime}(i=0,1, \ldots, m)$, and $Q_{i}(i=0,1, \ldots$, $m-2)$ lies below, and each of the points $P_{i}(i=2,3, \ldots, m)$, lies above the $x$-axis, while $P_{1}$ and $Q_{m-1}$ lie on the $x$-axis in the interval $(a, b), R_{2}$ changes sign exactly $(m-1)+(m-1)=2 m-2$ times, while $R_{3}$ changes sign exactly $(m-2)+(m-3)=2 m-5$ times. By $(*)$,

$$
H_{j}<h<H_{j-1} \Leftrightarrow[(n-1+2 j) / 2]<(b-a) / h<[(n+1+2 j) / 2],
$$

which implies $m=[(n-1+2 j) / 2]$, since

$$
[(n-1+2 j) / 2]+1=[(n+1+2 j) / 2] \text {. }
$$


If $j=2$, then $m=[(n+3) / 2]$, and since $n$ even, $n=2 m-2 ;$ if $j=3$, then $m=[(n+5) / 2]$, and since $n$ odd, $n=2 m-5$. Thus, $R_{2}$ and $R_{3}$ have the required number of $n$ changes of sign. Since polygonal arcs are continuous, $P_{0}=P_{0}(a, 0)$, and $Q_{m}=Q_{m}(b, 0), R_{2}$ and $R_{3}$ satisfy the conditions of the Theorem.

It remains to prove that $R_{2}$ and $R_{3}$ have no chords of length $h$ with endpoints in $(a, b)$. But because of the construction of these polygonal arcs, simple reasoning shows that $R_{2}(x+h)-R_{2}(x)=1$ for $x \in\left[b_{m}^{\prime}, b-h\right)$. If $x \in\left[a, b_{m}^{\prime}\right]$, the difference $R_{2}(x+h)-R_{2}(x)$ is strictly increasing from 0 to 1 , assuming these values just at the endpoints of $\left[a, b_{m}^{\prime}\right]$. Thus, $R_{2}(x+h)-$ $R_{2}(x) \neq 0$ for every $x \in(a, b-h)$. Quite alike, $R_{3}(x+h)-R_{3}(x)=1$ for $x \in\left[b_{m}, a_{m-1}\right]$. The difference $R_{3}(x+h)-R_{3}(x)$ is strictly increasing from 0 to 1 for $x \in\left[a, b_{m}\right]$, and strictly decreasing from 1 to 0 for $x \in\left[a_{m-1}\right.$, $b-h]$, assuming 0 only in $a$ and $b-h$. Hence, $R_{3}(x+h)-R_{3}(x) \neq 0$ for $x \in(a, b-h)$. This completes the proof of the Theorem.

What happens when $n=0$ (the case omitted in the above Theorem)? Items 1 and 2 of the proof hold for $n=0$ as well as for $n>0$, while in the third item, $n=0$ is impossible. In item 4 for $n=0$, the only case possible is $n^{*}=n$. Since Lemma 1 holds for $n=0$, we come to the following result.

Corollary. If $f$ satisfies the conditions of the Theorem, and $n=0$, then for every positive $h$ such that $h<(b-a) / 2$, there is a number $x \in(a$, $b-h)$ such that $f(x+h)=f(x)$.

We cannot improve this result, since there is a function $R$ satisfying the conditions of the Theorem and $R(x+h)-R(x) \neq 0$ for $h=(b-a) / 2$ and $x \in(a, b-h)$. If $h=(b-a) / 2$, then $a+h=b-h=(a+b) / 2$. Let $R((a+b) / 2)=0$. The function

$$
R(x)= \begin{cases}-(x-a)(x-(a+b) / 2) & \text { for } a \leq x \leq(a+b) / 2, \\ -2(x-b)(x-(a+b) / 2) & \text { for }(a+b) / 2 \leq x \leq b\end{cases}
$$

is continuous, $R(a)=R((a+b) / 2)=R(b)=0$, and does not change sign in $(a, b)$. If $x \in(a,(a+b) / 2)$, then $x+h \in((a+b) / 2, b)$. Since $h=(b-a) / 2$,

$$
R(x+h)-R(x)=-(x-(a+b) / 2)(x-a) \neq 0
$$

for $x \in(a,(a+b) / 2)$, and $R$ has no chords of length $(b-a) / 2$ with endpoints in $(a, b)$.

If throughout the foregoing, the condition of $n$ changes of sign is replaced by $n$ zeros in $(a, b)$, we come to the following results, whereof (1) has already been remarked by Levit. 
(1) For any $h \leq(b-a) /[(n+3) / 2]$ there is a number $x \in[a, b-b]$;

(2) for any $h \leq(b-a) /[(n+4) / 2]$ there is a number $x \in[a, b-h)$ $(x \in(a, b-b]$, respectively);

(3) for any $h \leq(b-a) /[(n+5) / 2]$ there is a number $x \in(a, b-h)$ such that $f(x)=f(x+h)$.

The bounds given above are again the best possible ones; $R_{2}$ can be directly used in (2) for $n$ odd and $x \in(a, b-h]$, and $R_{3}$ in (3) for $n$ odd. The other polygonal arcs can be constructed likewise.

\section{REFERENCE}

1. R. J. Levit, The finite difference extension of Rolle's theorem, Amer. Math. Monthly 70 (1963), 26-30. MR $26 \# 5107$

INSTITUT C FÜR MATHEMATIK, TECHNISCHE UNIVERSITÄT BRAUNSCHWEIG, 33 BRAUNSCHWEIG, POCKELSSTRASSE 14 (FORUM), FEDERAL REPUBLIC OF GERMANY 